\title{
Fourier Domain Optical Coherence Tomography for Retinal Imaging with 800-nm Swept Source: Real-time Resampling in $k$-domain
}

\author{
Sang-Won Lee*, Hyun-Woo Song, Bong-Kyu Kim, Moon-Youn Jung, and Seung-Hwan Kim \\ BT Convergence Research Department, IT Convergence Research Laboratory, \\ Electronics and Telecommunications Research Institute, Daejeon 305-700, Korea \\ Jae Du Cho and Chang-Seok Kim \\ Department of Cogno-Mechatronics Engineering, Pusan National University, Busan 609-735, Korea
}

(Received April 27, 2011 : revised August 17, 2011 : accepted August 18, 2011)

\begin{abstract}
In this study, we demonstrated Fourier-domain/swept-source optical coherence tomography (FD/SS-OCT) at a center wavelength of $800 \mathrm{~nm}$ for in vivo human retinal imaging. A wavelength-swept source was constructed with a semiconductor optical amplifier, a fiber Fabry-Perot tunable filter, isolators, and a fiber coupler in a ring cavity. Our swept source produced a laser output with a tuning range of $42 \mathrm{~nm}(779$ to $821 \mathrm{~nm}$ ) and an average power of $3.9 \mathrm{~mW}$. The wavelength-swept speed in this configuration with bidirectionality is 2,000 axial scans per second. In addition, we suggested a modified zero-crossing method to achieve equal sample spacing in the wavenumber $(k)$ domain and to increase the image depth range. FD/SS-OCT has a sensitivity of $\sim 89.7 \mathrm{~dB}$ and an axial resolution of $10.4 \mu \mathrm{m}$ in air. When a retinal image with 2,000 A-lines/frame is obtained, an acquisition speed of $2.0 \mathrm{fps}$ is achieved.
\end{abstract}

Keywords : Optical coherence tomography, Swept source, Retina, Ophthalmology

OCIS codes : (170.0170) Medical optics and biotechnology; (110.4500) Optical coherence tomography;

(170.4500) Optical coherence tomography; (170.4470) Ophthalmology

\section{INTRODUCTION}

Optical coherence tomography (OCT) has been extensively studied over the past 20 years, as it provides in vivo real-time cross-sectional images and three-dimensional volumetric images of biological tissues with a high axial resolution [1]. In ophthalmology, OCT was already commercialized and widely used for non-invasive structural and quantitative imaging of the human retina and anterior segment [2-5]. Recently, Fourier-domain OCT (FD-OCT), which includes a spectral-domain method and a swept-source method, has become a preferred method owing to its superior features of higher acquisition speed and sensitivity [6-10]. Due to the limited number of camera pixels, spectral-domain OCT (FD/SD-OCT) has trade-offs among the axial resolution, imaging depth range, acquisition time, and sensitivity roll-off $[11,12]$. FD-OCT based on a swept source (FD/SS-OCT) has overcome these trade-offs. FD/SS-OCT can obtain a low sensitivity roll-off as it realizes a long imaging depth and reduced fringe washout. In addition, FD/SS-OCT can be used to remove the $\mathrm{DC}$ autocorrelation noise by employing a dual-balanced detector. Finally, a faster acquisition speed is possible since the rise time of a photodiode is faster than that of cameras based on charge-coupled device (CCD) and complementary metal-oxide semiconductor (CMOS) chips [13-15].

Although FD/SS-OCT has these advantages, it has been studied, only by a few groups, at $840 \mathrm{~nm}$ or $850 \mathrm{~nm}$, which is more useful than $\mathrm{FD} / \mathrm{SS}-\mathrm{OCT}$ at $1310 \mathrm{~nm}$ with regard to retina imaging, because the characteristics of optical components in the $800-\mathrm{nm}$ region have limitations. Recently, a swept source in the $1.0 \mu \mathrm{m}$ region has been actively used for retina imaging because water absorption has a local minimum and the dispersion in water is near zero in the $1.0 \mu \mathrm{m}$ region [16, 17]. However, a broadband source centered in the $800 \mathrm{~nm}$ region rather than in the $1.0 \mu \mathrm{m}$ region is favorable for obtaining high resolution [18]. Chinn et al. [19] have developed an 840-nm swept source

\footnotetext{
*Corresponding author: swlee76@etri.re.kr

Color versions of one or more of the figures in this paper are available online.
} 
with a full-width at half-maximum (FWHM) of $20 \mathrm{~nm}$, which utilizes a superluminescent light-emitting diode and a Littrow configuration with a grating and a resonant galvanometer scanner. Lim et al. [20, 21], Lee et al. [22], and Srinivasan et al. [17] introduced an 850-nm swept source with an FWHM over $35 \mathrm{~nm}$ employing semiconductor optical amplifiers (SOAs). Lim et al. developed a swept source at $850 \mathrm{~nm}$ based on a ring cavity including a free-space isolator [20] or a linear cavity with only an optical fiber coupler (fiber loop mirror) [21]. They used the spectral filter comprised of a diffractive grating, achromatic lenses, and a polygon mirror. Lee et al. and Srinivasan et al. also constructed spectral filters on the basis of Littman and Littrow configurations, respectively $[17,22]$. These configurations included a diffractive grating and galvanometer scanner. Although these 850-nm swept sources had a broad spectrum full bandwidth of approximately $60 \mathrm{~nm}$, these did not have the isobestic point, for which the hemoglobin $(\mathrm{Hb})$ and oxygenated hemoglobin $\left(\mathrm{HbO}_{2}\right)$ absorption coefficients are equal [23, 24], However, $800-\mathrm{nm}$ wavelength is very close to the isobestic point. Therefore, the spectroscopic OCT using 800-nm swept source instead of $840-\mathrm{nm}$ or $850-\mathrm{nm}$ swept source can measure functional information such as hemoglobin oxygen saturation level $\left(\mathrm{SO}_{2}\right)$ [23] in retina blood vessels. The spectroscopic OCT can provide spectroscopic properties and biochemical properties of biological tissue as well as a cross-sectional image and hemoglobin oxygen saturation level [25, 26].

In this preliminary study, we demonstrate FD/SS-OCT using an 800-nm swept source for in vivo human retina imaging. Previous swept sources based on gratings (Littman and Littrow configurations) have a bulky type filter with free space path. Free space path in the swept source sometimes causes large insertion loss, which is a hurdle for achieving a high optical power. Very recently, a fiber Fabry-Perot tunable filter, a fiber-coupled isolator, and an SOA at a center wavelength of $800 \mathrm{~nm}$ were commercialized. Therefore, we could easily construct the swept source based on a fiber type ring cavity design without free space path, thus securing low insertion loss. The tuning range of the swept source was from 779-821 nm, and the averaged optical power was approximately $3.9 \mathrm{~mW}$. The wavelength-swept speed in this configuration operating in bidirectional mode is 2,000 axial scans per second. Additionally, in this paper, we suggest a modified zero-crossing method to achieve equal sample spacing in the wavenumber $(k)$ domain. The retina of a healthy volunteer was measured, with an A-line rate of $2.0 \mathrm{kHz}$, an axial resolution of $10.4 \mu \mathrm{m}$ in air $(7.6$ $\mu \mathrm{m}$ in tissues), and a sensitivity of $89.7 \mathrm{~dB}$.

\section{SWEPT SOURCE SETUP AND CHARACTERIZATION}

Figure 1 shows a schematic of a conventional ring-cavity swept source at the center wavelength of $800 \mathrm{~nm}$. We

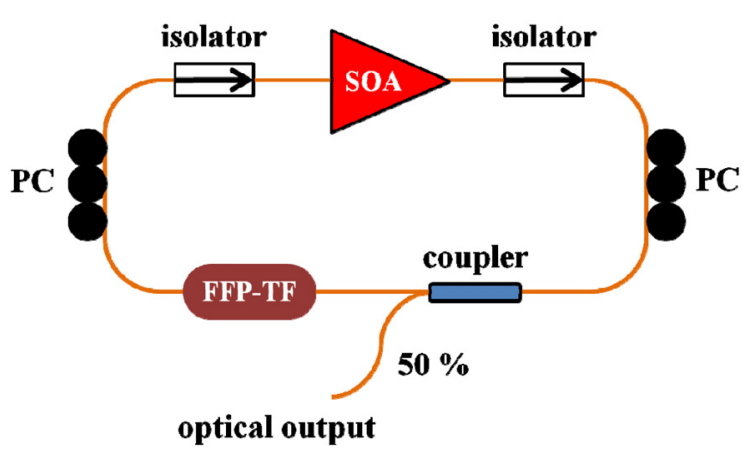

FIG. 1. Schematic of the wavelength-swept source with a conventional ring cavity at $800 \mathrm{~nm}$. FFP-TF: Fiber Fabry-Perot tunable filter, PC: polarization controller.

constructed a swept source with recently commercialized fiberbased isolators (Opto-Link Corp. Ltd.) and a Fabry-Perot tunable filter (FFP-TF2, Micron Optics). The gain medium is a fiber-coupled SOA (SOA-372-800, Superlum Diodes Ltd.). The SOA has a $-3 \mathrm{~dB}$ optical gain bandwidth of 20 $\mathrm{nm}$. The SOA was coupled with isolators for a unidirectional ring cavity as shown in Fig. 1. Half of the total optical power fed from the $1 \times 2$ fiber coupler is amplified by the SOA at a specific wavelength filtered by the FFP-TF. The filtered light was amplified by the SOA. The other port $(50 \%)$ of the fiber coupler was used for the output of amplified light. When the FFP-TF was driven using a $1.0 \mathrm{kHz}$ triangular waveform, the swept source achieved an effective bidirectional sweep rate of $2.0 \mathrm{kHz}$. The SOA was supplied with a current of $160 \mathrm{~mA}$.

Figure 2 (a) is a peak-held spectrum of the swept source measured by an optical spectrum analyzer. This swept source has a center wavelength of $802.3 \mathrm{~nm}$ with an average power of $3.5 \mathrm{~mW}$ and full scanning range of $42 \mathrm{~nm}(779 \mathrm{~nm}$ to $821 \mathrm{~nm}$ ). Figure 2 (b) shows the output trace of the swept source in the time domain. Because the optical power and shape of the swept source are dependent on the polarization state in the cavity and on the birefringence of components, the polarization state is controlled by a polarization controller [27]. As shown in Fig. 2 (b), a higher scanning speed of greater than $2.0 \mathrm{kHz}$ causes a significant power difference between the forward scan and the backward scan because of nonlinearity of the gain medium [27-30]. The power of the forward scan is higher than that of the backward scan. Figure 3 (a) and (b) show the point spread functions (PSFs) for various path length differences between the two arms when the wavelength is forwardscanned and backward-scanned, respectively. We measured the maximum imaging depth range $(\Delta z)$ to be approximately $6.9 \mathrm{~mm}$ while sampling points were being increased and the sample mirror was being moved. Therefore, the instantaneous spectral bandwidth could be calculated to be $0.023 \mathrm{~nm}$ by the equation of $\Delta z=\lambda_{\mathrm{o}}^{2} / 4 \delta \lambda$, where $\lambda_{\mathrm{o}}$ is the center wavelength and $\delta \lambda$ is an instantaneous spectral bandwidth. The filter line-width of the FFP-TF is $0.02 \mathrm{~nm}$, 


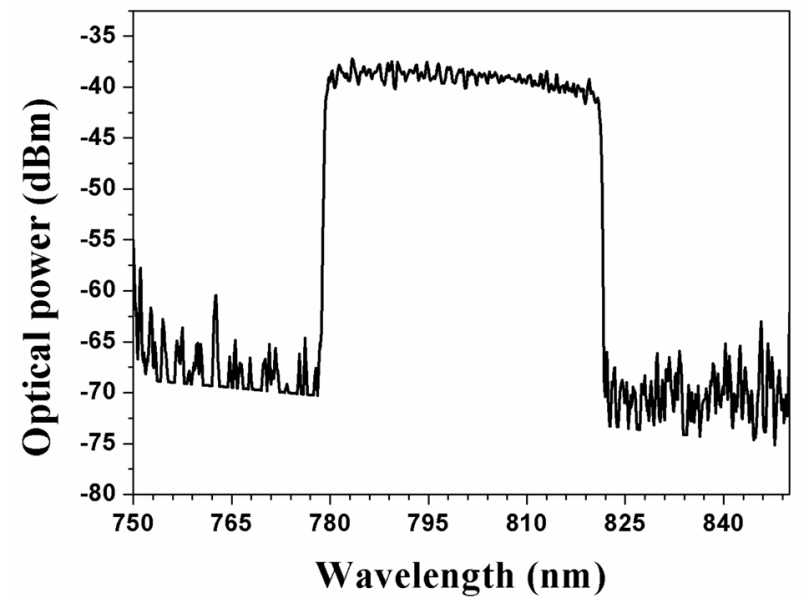

(a)

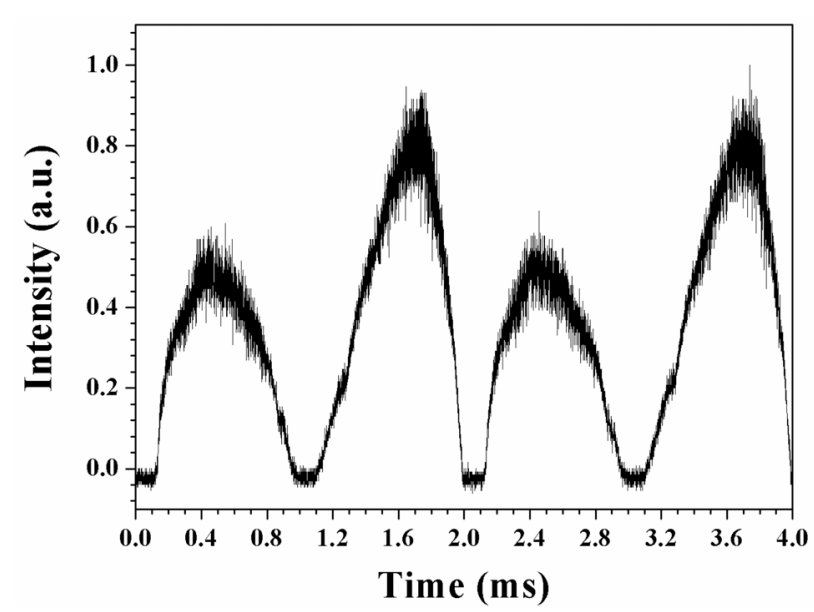

(b)

FIG. 2. Optical spectra of the swept source. (a) Peak-held spectrum with the center wavelength of $800 \mathrm{~nm}$. (b) The swept source output trace in the time domain when the filter is scanned at $1.0-\mathrm{kHz}$ triangular waveform.

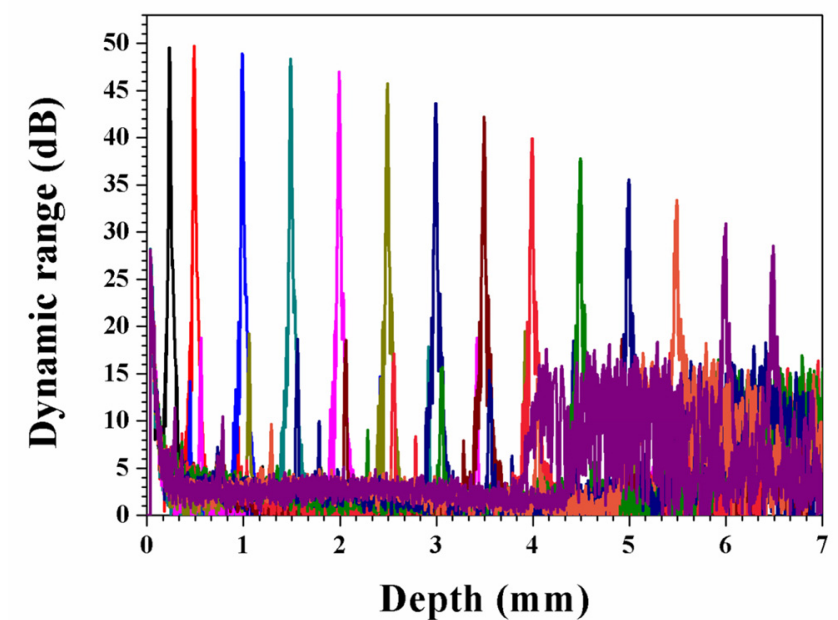

(a)

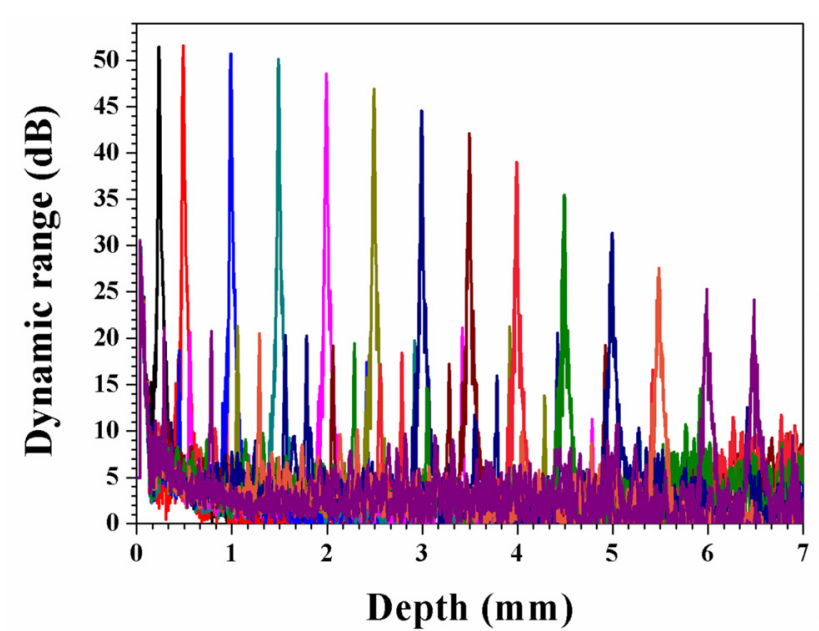

(b)

FIG. 3. The point spread functions (PSFs) for various imaging depths when the wavelength is (a) forward-scanned and (b) backward-scanned. The maximum imaging depth range of approximately $6.9 \mathrm{~mm}$ is measured. Therefore, an instantaneous spectral linewidth could be calculated to $0.023 \mathrm{~nm}$.

according to the manufacturing company. Therefore, the calculated instantaneous spectral bandwidth was a reasonable spectral line-width value.

\section{FD/SS-OCT SETUP AND DATA PROCESSING}

We constructed the FD/SS-OCT based on a Michelson interferometer for retinal imaging as shown in Fig. 4 (a). Light from the swept source was incident onto a MachZehnder interferometer module (INT-MZI-850, Thorlabs Inc.). This Mach-Zehnder interferometer module was constructed from a 95:5 optical fiber coupler, three 50:50 optical fiber couplers, and a balanced photodiode as shown in small box of Fig 4 (a), which is a schematic provided by the manufacturing company. In the Mach-Zehnder interferometer module, $5 \%$ of the light from the swept source was used for the interference signal and power monitor. An output port of the Mach-Zehnder interferometer module gave out $95 \%$ of the light of the swept source and was coupled with an optical circulator. Light from the MachZehnder interferometer module and the optical circulator was incident on a $2 \times 2$ optical fiber coupler and was split into reference and sample arms with 50:50 ratios. The sample arm consists of 2-D galvanometers with silver-coated mirrors (TS8203, Beijing Century Sunny Technology Co., Ltd), an achromatic doublet lens $(f=30$, Thorlabs Inc.), and a double aspheric ocular lens (40 D, Volk Optical Inc.) as shown in Fig. 4 (a). The 2-D galvanometers were controlled 


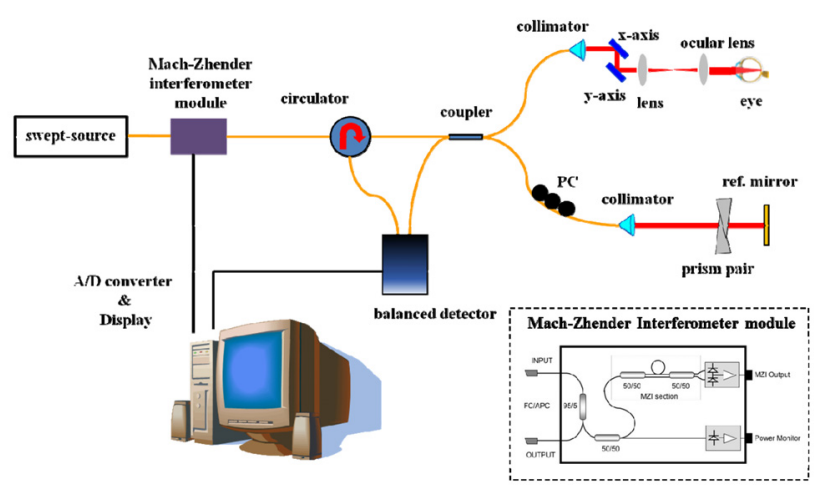

(a)

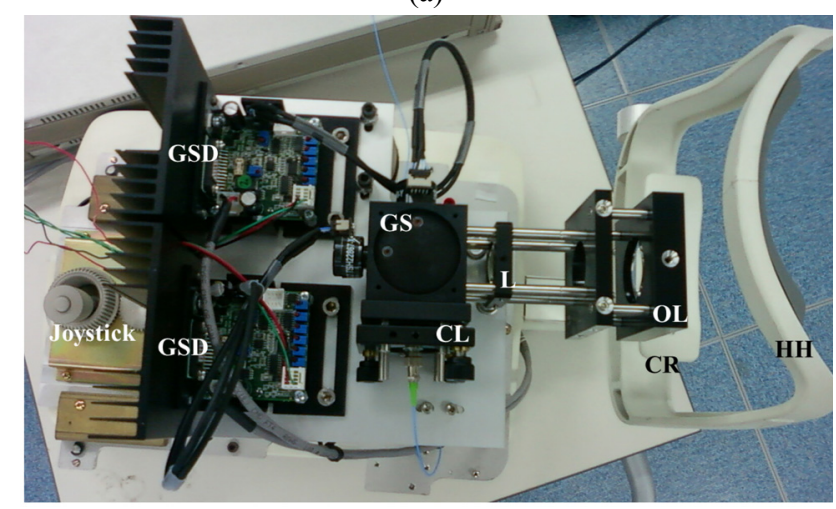

(b)

FIG. 4. Schematic and photo of the FD/SS-OCT system for retinal imaging. (a) schematic of the FD/SS-OCT system. PC: polarization controller. (b) photo of the sample arm in the FD/SS-OCT system. GSD: galvano scanner driver, GS: 2D galvano scanner, CL: collimation lens, L: achromatic doublet lens, OL: ocular lens, CR: chin rest, HH: head holder.

by an analog-output board (PCI-6722, National Instruments Corp.). To correct dispersion differences between the reference and sample arms, we used a dispersion compensation prism pair (AFS-SF10, Thorlabs Inc.) in the reference arm. We used a neutral density (ND) filter in the reference arm to reduce the source intensity noise [31]. Recombined light from the reference and sample arms was incident onto the balanced detector (PDB110A, Thorlabs Inc.) via the circulator and coupler. The interference signals from both the Mach-Zehnder interferometer module and the Michelson interferometer were converted by a high-speed digitizer with a 12-bit, 200-MS/s, and 256-MB onboard memory (PCI5124, National Instruments Corp.). 2,000 samples were acquired per A-scan and per channel of the digitizer at 2.0 $\mathrm{kHz}$. Figure 4 (b) is a photo of the sample arm at our FD/SS-OCT system for retinal imaging. We used the frame of a keratometer, including joystick, chin rest and head holder. The sample arm was put on the frame of the keratometer as shown in Fig. 4 (b).

Since the FFP-TF was driven by a triangular function, we performed numerical resampling of the raw fringe data by using the zero-crossing method to achieve equal sampling
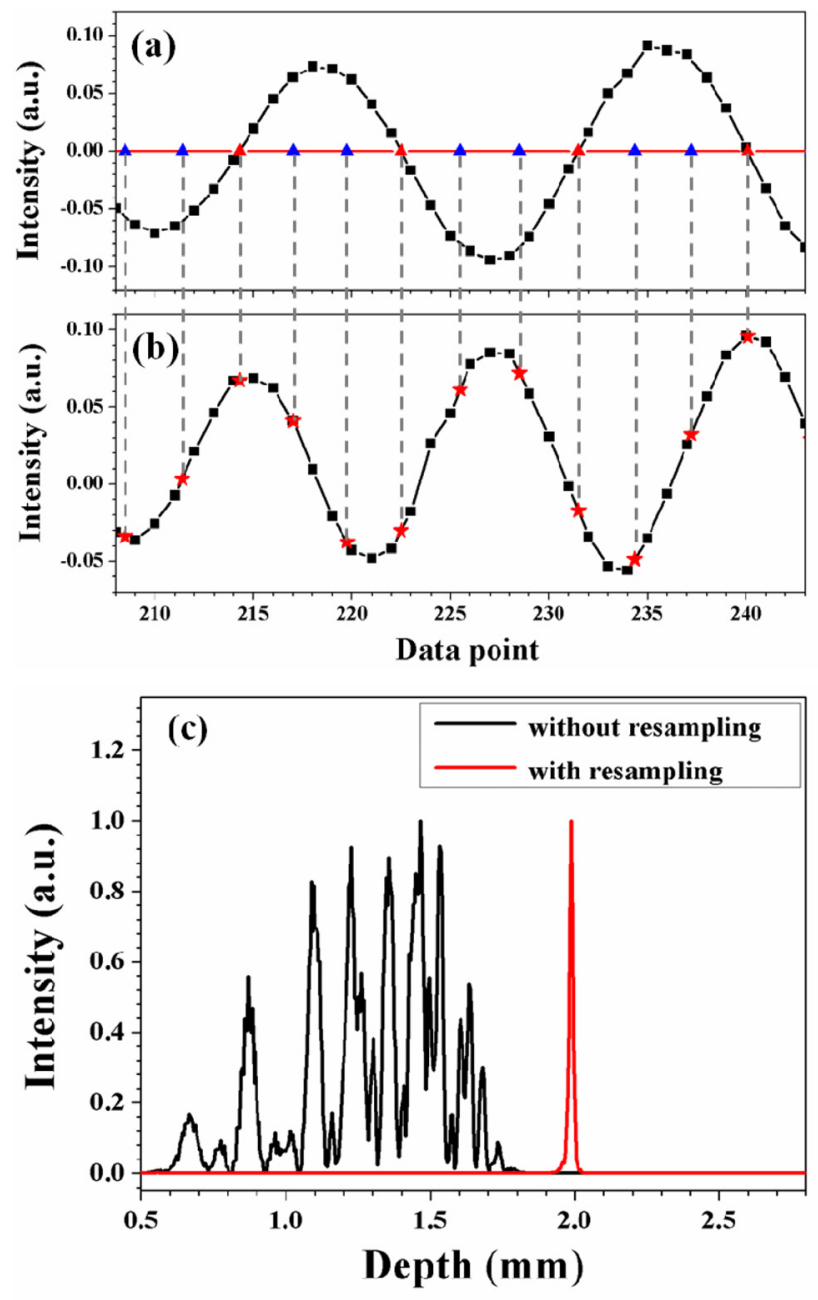

FIG. 5. Modified zero-crossing method for resampling in the k-domain. (a) zero-crossing points (red triangles) and two points (blue triangles) between the two zero-crossing points in the Mach-Zehnder interference signal, (b) resampled data (red stars) in Michelson interference signal, and (c) point spread functions after Fourier transform without and with resampling.

space in the $k$-domain. Figure 5 (a) shows the interference signal (black rectangle) from the Mach-Zehnder interferometer module. First, the zero-crossing points (red triangle) in the interference signal were founded by the linear interpolation method, as shown in Fig. 5 (a). The total number of zero-crossing points was 384, and if only these points were used, the imaging depth range would be limited to approximately $1.45 \mathrm{~mm}$. Therefore, we modified the zerocrossing method to increase the number of sampling points and to obtain, consequently, long imaging depth range. After finding the zero-crossing points, two or three points between the two zero positions were selected, and the Michelson interference signal was resampled at each of these points. The intensity values at the resampled positions were obtained by linear interpolation. For example, when the zero positions 
(red triangles) and two points (blue triangles) between the two zero positions were selected as shown in Fig. 5 (a), the intensity values (red stars) at the resampled data points of the Michelson interference signal were calculated, as shown in Fig. 5 (b). Therefore, the imaging depth range was increased to approximately $4.3 \mathrm{~mm}$. Finally, the data was zero-filled so that the total number of data points was 2048. When a mirror was used as a sample, Fig. 5 (c) shows point spread functions at the same position after Fourier transformation both without and with resampling.

\section{RESULTS AND DISCUSSION}

An averaged optical power of $0.7 \mathrm{~mW}$ or less in the sample arm was incident onto a sample in accordance with safe ocular exposure limits set by the American National Standards Institute (ANSI) [32]. We used a $-35.3 \mathrm{~dB}$ partially reflecting mirror as the sample to measure performance of the FD/SS-OCT system. The dynamic range of our OCT system for forward and backward wavelength-sweep is shown in Fig. 3 (a) and (b), respectively. A decrease of 6 $\mathrm{dB}$ was observed within the depth range of approximately $3.0 \mathrm{~mm}$. It was found that the sensitivity of the system, which was determined by adding the sample attenuation constant $(35.3 \mathrm{~dB})$, was approximately $89.7 \mathrm{~dB}$ at a depth of $0.25 \mathrm{~mm}$. The axial resolution was measured to be 10.4 $\mu \mathrm{m}$ in air, corresponding to $7.6 \mu \mathrm{m}$ in tissue. Figure 6 shows axial resolution variations as a function of the depth. The measured axial resolution increased beyond $2.0 \mathrm{~mm}$. This degradation indicates that the interpolation process is sensitive to small errors at high fringe frequencies [8, 28]. When a 1,000 A-lines/frame image was obtained, an acquisition speed of 2.0 frames per second (fps) was achieved.

Figure 7 shows cross-sectional retinal images of a healthy volunteer. Figure 7 (a) was obtained without dispersion compensation. Figure 7 (b) is a dispersion compensated retinal image, but resampling was not yet carried out. Neither image

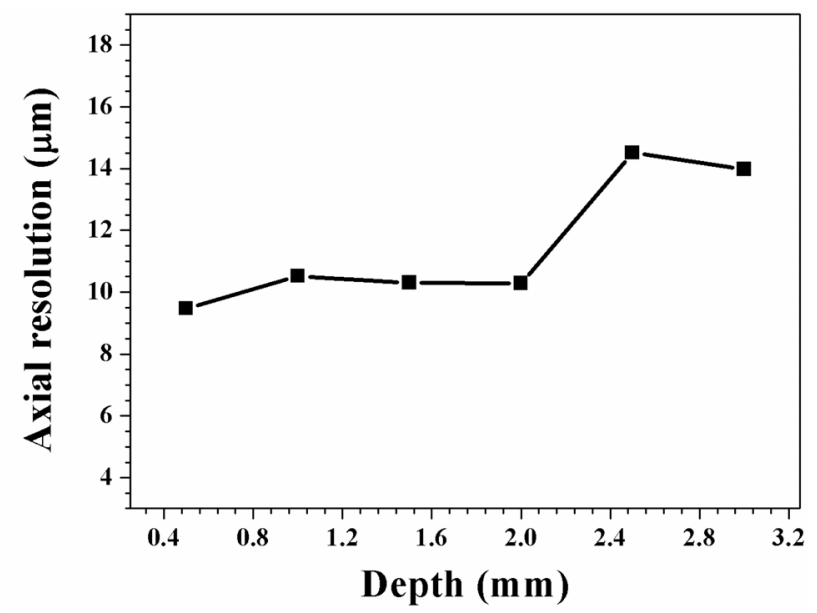

FIG. 6. Axial resolution variation as a function of the depth.

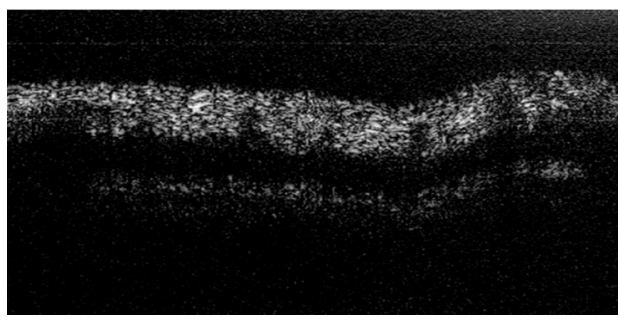

(a)

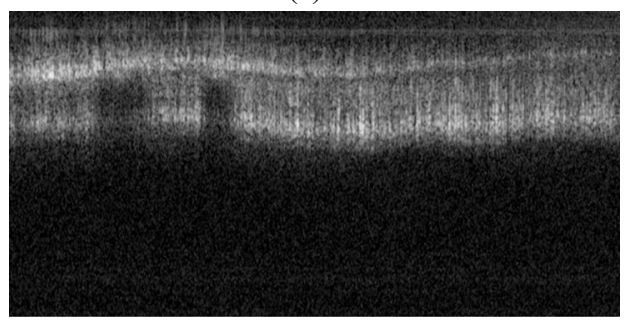

(b)

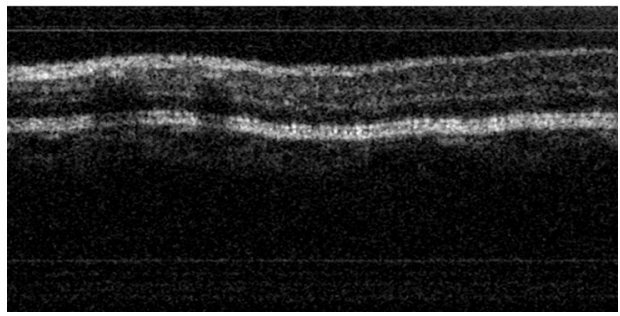

(c)

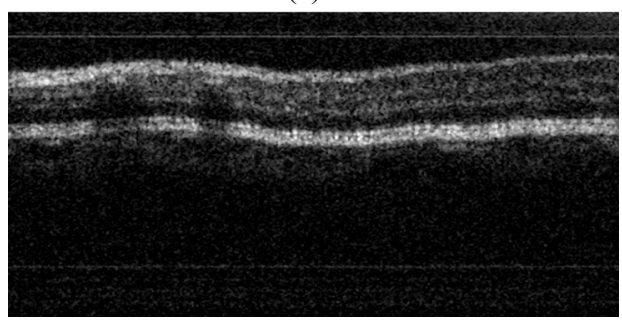

(d)

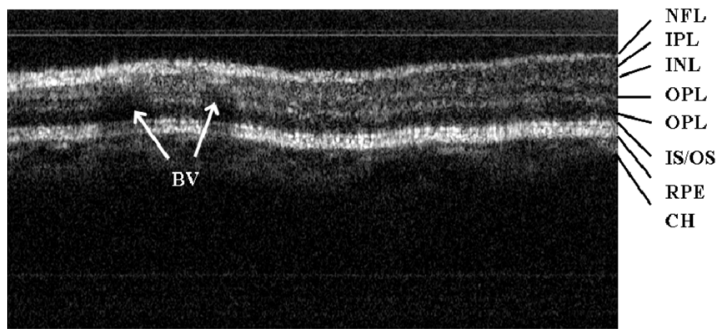

(e)

FIG. 7. Retinal images of a healthy volunteer. (a) OCT image with resampling and without dispersion compensation, (b) OCT image without resampling and with dispersion compensation, (c) OCT image with resampling and dispersion compensation using only forward wavelength-swept, (d) OCT image with resampling and dispersion compensation using only backward wavelength-swept, (e) OCT image with resampling and dispersion compensation using both wavelength-swept; NFL: nerve fiber layer, IPL: inner plexiform layer, INL: inner nuclear layer, OPL: outer plexiform layer, ONL: outer nuclear layer, IS/OS: photoreceptor inner segment/outer segment junction, RPE: retinal pigment epithelium, $\mathrm{CH}$ : choroid, BV: blood vessel. 
could present clear structural information of the retina. After applying both dispersion compensation and resampling to the original image, detailed structure of the retina was clearly distinguishable. Our swept source is continuously tuned with forward and backward wavelength directions because the swept source is driven by a triangular wave function. We obtained 2,000 lines per frame at both forward and backward wavelength directions and then chose 1,000 lines per frame at each wavelength direction. Figure 7 (c) and (d) were obtained at each swept directions, one is for the forward direction and the other is for the backward direction. We applied a median filter with $3 \times 3$ matrix to eliminate speckle noise and selected a region of interest (ROI) with an area of 1,000 (lateral) $\times 355$ (axial) pixels corresponding to a physical size of approximately $5.0 \mathrm{~mm}$ (width) $\times 1.5 \mathrm{~mm}$ (depth) in air. Figure 7 (e) shows the OCT image using both wavelength-swept directions. Therefore, the pixel size of Fig. 7 (e) is 2,000 (lateral) $\times 355$ (axial) pixels corresponding to a physical size of approximately $5.0 \mathrm{~mm}$ (width) $\times 1.5 \mathrm{~mm}$ (depth) in air.

$\mathrm{FD} / \mathrm{SS}-\mathrm{OCT}$ for retinal imaging at the 850 -nm region has been already studied by Lim et al. [24] and Srinivasan et al. [18]. This study showed preliminary results for highresolution and high-speed FD/SS-OCT in ophthalmology. In this study, our swept source still has limitations of the wavelength-tuning range and speed. In the previous papers [24], the wavelength-tuning ranges were $55 \mathrm{~nm}$ in the 850-nm region and the FWHMs were broader than our swept source. Although the wavelength-tuning range of our swept source is narrow $(42 \mathrm{~nm})$, the axial resolution (10.4 $\mu \mathrm{m})$ of our OCT system is lower than that of the previous result $(13 \mu \mathrm{m})$ because the center wavelength of our swept source is lower according to the equation: $\delta \mathrm{Z}=0.44 \times \lambda_{0}{ }^{2} / \Delta \lambda$, where $\Delta \lambda$ is the FWHM of the source. Currently, we are improving the wavelength-tuning range and speed of the swept source. Limitation of the wavelength-tuning range can be overcome by using multiple SOAs [33]. Increase of the wavelength-tuning range will also affect the axial resolution of an OCT system. Finally, it is easy to improve the limit of the wavelength-swept speed to hundreds of kilohertz if the cavity length is very short and it is combined with a high-speed filter of kilohertz order such as a polygon scanner or microelectromechanical system (MEMS) scanner [34-36].

\section{CONCLUSION}

In this study, we devised a wavelength-swept source at a center wavelength of $800 \mathrm{~nm}$ and developed an FD/SSOCT system for retinal imaging. The swept source was constructed using a ring cavity with an SOA, an FFP-TF, isolators, and a $1 \times 2$ fiber coupler (50:50 ratios). Our swept source produces laser output with a tuning range of $42 \mathrm{~nm}(779$ to $821 \mathrm{~nm})$ and an average power of $3.9 \mathrm{~mW}$. The wavelength-swept speed in this configuration with bidirectionality is 2,000 axial scans per second. In addition, the instantaneous spectral bandwidth was calculated to be $0.023 \mathrm{~nm}$. We suggested a modified zero-crossing method to achieve equal sample spacing in the $k$-domain and to increase an image depth range. Our FD/SS-OCT has a sensitivity of $\sim 89.7 \mathrm{~dB}$ and an axial resolution of $10.4 \mu \mathrm{m}$ in air. We observed a decrease of $6 \mathrm{~dB}$ within a depth range of approximately $3.0 \mathrm{~mm}$. When a 1,000 A-lines/frame retinal image was obtained, an acquisition speed of $2.0 \mathrm{fps}$ was achieved. Finally, we were able to obtain in vivo a cross-sectional retinal image of a healthy volunteer. Currently, we are developing a wide-band (> FWHM of $100 \mathrm{~nm}$ ) and high-speed $(>40 \mathrm{kHz})$ swept source with multi SOAs at 830-nm region.

\section{ACKNOWLEDGMENT}

This work was supported by the ETRI Support Program of the Ministry of Knowledge Economy (10035602). This work was also supported by the National Research Foundation of Korea Grant funded by the Korean Government (NRF-2009-352-D00349).

\section{REFERENCES}

1. D. Huang, E. A. Swanson, C. P. Lin, J. S. Schuman, W. G. Stinson, W. Chang, M. R. Hee, T. Flotte, K. Gregory, C. A. Puliafito, and J. G. Fujimoto, "Optical coherence tomography," Science 254, 1178-1181 (1991).

2. C. A. Puliafito, M. R. Hee, C. P. Lin, E. Reichel, J. S. Schuman, J. S. Duker, J. A. Izatt, E. A. Swanson, and J. G. Fujimoto, "Imaging of macular diseases with optical coherence tomography," Ophthalmology 102, 217-229 (1995).

3. J. S. Schuman, M. R. Hee, A. V. Arya, T. Pedut-Kloizman, C. A. Puliafito, J. G. Fujimoto, and E. A. Swanson, "Optical coherence tomography: a new tool for glaucoma diagnosis," Curr. Opin. Ophthalmol. 6, 89-95 (1995).

4. J. S. Schuman, M. R. Hee, C. A. Puliafito, C. Wong, T. Pedut-Kloizman, C. P. Lin, E. Hertzmark, J. A. Izatt, E. A. Swanson, and J. G. Fujimoto, "Quantification of nerve fiber layer thickness in normal and glaucomatous eyes using optical coherence tomography," Arch. Ophthalmol. 113, 586-596 (1995).

5. J. A. Izatt, M. R. Hee, E. A. Swanson, C. P. Lin, D. Huang, J. S. Schuman, C. A. Puliafito, and J. G. Fujimoto, "Micrometer-scale resolution imaging of the anterior eye in vivo with optical coherence tomography," Arch. Ophthalmol. 112, 1584-1589 (1994).

6. R. Leitgeb, C. Hitzenberger, and A. Fercher, "Performance of fourier domain vs. time domain optical coherence tomography," Opt. Express 11, 889-894 (2003).

7. M. Choma, M. Sarunic, C. Yang, and J. Izatt, "Sensitivity advantage of swept source and Fourier domain optical coherence tomography," Opt. Express 11, 2183-2189 (2003).

8. S. Yun, G. Tearney, B. Bouma, B. Park, and J. de Boer, "High-speed spectral-domain optical coherence tomography 
at $1.3 \mu \mathrm{m}$ wavelength," Opt. Express 11, 3598-3604 (2003).

9. B. White, M. Pierce, N. Nassif, B. Cense, B. Park, G. Tearney, B. Bouma, T. Chen, and J. de Boer, "In vivo dynamic human retinal blood flow imaging using ultra-highspeed spectral domain optical Doppler tomography," Opt. Express 11, 3490-3497 (2003).

10. Y. Yasuno, V. D. Madjarova, S. Makita, M. Akiba, A. Morosawa, C. Chong, T. Sakai, K. Chan, M. Itoh, and T. Yatagai, "Three-dimensional and high-speed swept-source optical coherence tomography for in vivo investigation of human anterior eye segments," Opt. Express 13, 10652-10664 (2005).

11. M. Wojtkowski, V. Srinivasan, T. Ko, J. Fujimoto, A. Kowalczyk, and J. Duker, "Ultrahigh-resolution, high-speed, Fourier domain optical coherence tomography and methods for dispersion compensation," Opt. Express 12, 2404-2422 (2004).

12. S.-W. Lee, H.-W. Jeong, B.-M. Kim, Y.-C. Ahn, W. Jung, and Z. Chen, "Optimization for axial resolution, depth range, and sensitivity of spectral domain optical coherence tomography at $1.3 \mu \mathrm{m}$," J. Korean Phys. Soc. 55, 2354-2360 (2009).

13. B. Potsaid, B. Baumann, D. Huang, S. Barry, A. E. Cable, J. S. Schuman, J. S. Duker, and J. G. Fujimoto, "Ultrahigh speed $1050 \mathrm{~nm}$ swept source/Fourier domain OCT retinal and anterior segment imaging at 100,000 to 400,000 axial scans per second," Opt. Express 18, 20029-20048 (2010).

14. W. Wieser, B. R. Biedermann, T. Klein, C. M. Eigenwillig, and R. Huber, "Multi-megahertz OCT: high quality 3D imaging at 20 million A-scans and 4.5 GVoxels per second," Opt. Express 18, 14685-14704 (2010).

15. T. Klein, W. Wieser, C. M. Eigenwillig, B. R. Biedermann, and R. Huber, "Megahertz OCT for ultrawide-field retinal imaging with a $1050 \mathrm{~nm}$ Fourier domain mode-locked laser," Opt. Express 19, 3044-3062 (2011).

16. B. Považay, K. Bizheva, B. Hermann, A. Unterhuber, H. Sattmann, A. Fercher, W. Drexler, C. Schubert, P. Ahnelt, M. Mei, R. Holzwarth, W. Wadsworth, J. Knight, and P. St. J. Russell, "Enhanced visualization of choroidal vessels using ultrahigh resolution ophthalmic OCT at $1050 \mathrm{~nm}$," Opt. Express 11, 1980-1986 (2003).

17. V. J. Srinivasan, D. C. Adler, Y. Chen, I. Gorczynska, R. Huber, J. S. Duker, J. S. Schuman, and J. G. Fujimoto, "Ultrahigh-speed optical coherence tomography for threedimensional and en face imaging of the retina and optic nerve head," Invest. Ophthalmol. Vis. Sci. 49, 5103-5110 (2008).

18. V. J. Srinivasan, R. Huber, I. Gorczynska, J. G. Fujimoto, J. Y. Jiang, P. Reisen, and A. E. Cable, "High-speed, highresolution optical coherence tomography and retinal imaging with a frequency-swept laser at $850 \mathrm{~nm}$," Opt. Lett. 32, 361-363 (2007).

19. R. S. Chinn, E. A. Swanson, and J. G. Fujimoto, "Optical coherence tomography using a frequency-tunable optical source," Opt. Lett. 22, 340-342 (1997).

20. H. Lim, J. F. de Boer, B. H. Park, E. C. Lee, R. Yelin, and S. H. Yun, "Optical frequency domain imaging with a rapidly swept laser in the $815-870 \mathrm{~nm}$ range," Opt. Express 14, 5937-5944 (2006).
21. H. Lim, M. Mujat, C. Kerbage, E. C. Lee, Y. Chen, T. C. Chen, and J. F. de Boer, "High-speed imaging of human retina in vivo with swept-source optical coherence tomography," Opt. Express 14, 12902-12908 (2006).

22. S.-W. Lee, C.-S. Kim, and B.-M. Kim, "External line-cavity wavelength-swept source at $850 \mathrm{~nm}$ for optical coherence tomography," IEEE Photon. Technol. Lett. 19, 176-178 (2007).

23. D. J. Faber, E. G. Mik, M. C. G. Aalders, and T. G. van Leeuwen, "Light absorption of (oxy-)hemoglobin assessed by spectroscopic optical coherence tomography," Opt. Lett. 28, 1436-1438 (2003).

24. J. U. Kang, J.-H. Han, X. Liu, and K. Zhang, "Commonpath optical coherence tomography for biomedical imaging and sensing," J. Opt. Soc. Korea 14, 1-13 (2010).

25. U. Morgner, W. Drexler, F. X. Kärtner, X. D. Li, C. Pitris, E. P. Ippen, and J. G. Fujimoto, "Spectroscopic optical coherence tomography," Opt. Lett. 25, 111-113 (2000).

26. C. Xu, J. Ye, D. L. Marks, and S. A. Boppart, "Nearinfrared dyes as contrast-enhancing agents for spectroscopic optical coherence tomography," Opt. Lett. 29, 1647-1649 (2004).

27. S. H. Yun, C. Boudoux, M. C. Pierce, J. F. de Boer, G. J. Tearney, and B. E. Bouma, "Extended-cavity semiconductor wavelength-swept laser for biomedical imaging," IEEE Photon. Technol. Lett. 16, 293-295 (2004).

28. R. Huber, M. Wojtkowski, K. Taira, J. Fujimoto, and K. Hsu, "Amplified, frequency swept lasers for frequency domain reflectometry and OCT imaging: design and scaling principles," Opt. Express 13, 3513-3528 (2005).

29. A. Bilenca, S. H. Yun, G. J. Tearney, and B. E. Bouma, "Numerical study of wavelength-swept semiconductor ring lasers: the role of refractive-index nonlinearities in semiconductor optical amplifiers and implications for biomedical imaging applications," Opt. Lett. 31, 760-762 (2006).

30. M. Jeon, J. Zhang, and Z. Chen, "Characterization of Fourier domain mode-locked wavelength swept laser for optical coherence tomography imaging," Opt. Express 16, 3727-3737 (2008).

31. W. V. Sorin and D. M. Baney, "A simple intensity noise reduction technique for optical low-coherence reflectometry," IEEE Photon. Technol. Lett. 4, 1404-1406 (1992).

32. A. N. S. Institute, "American national standard for safe use of lasers," in ANSI Z 136-1 (2000).

33. M. Y. Jeon, J. Zhang, Q. Wang, and Z. Chen, "High-speed and wide bandwidth Fourier domain mode-locked wavelength swept laser with multiple SOAs," Opt. Express 16, 2547-2554 (2008).

34. W. Y. Oh, B. J. Vakoc, M. Shishkov, G. J. Tearney, and B. E. Bouma, " $>400 \mathrm{kHz}$ repetition rate wavelength-swept laser and application to high-speed optical frequency domain imaging," Opt. Lett. 35, 2919-2921 (2010).

35. M. Kuznetsov, W. Atia, B. Johnson, and D. Flanders, "Compact ultrafast reflective Fabry-Perot tunable lasers for OCT imaging applications," Proc. SPIE 7554, 75541F (2010).

36. K. Totsuka, K. Isamoto, T. Sakai, A. Morosawa, and C. Chong, "MEMS scanner based swept source laser for optical coherence tomography," Proc. SPIE 7554, 75542Q (2010). 